\title{
Testing Exponentiality Against UBAC Using Kernel Methods
}

\author{
M. M. Mohie El-Din ${ }^{1}$, S. E. Abu-Youssef ${ }^{1}$, M. KH. Hassan ${ }^{2, *}$ \\ ${ }^{1}$ Department of Mathematics, Faculty of Science, \\ Al- Azhar University, Cairo, Egypt; \\ ${ }^{2}$ Department of Mathematics, Faculty of Education, \\ Ain Shams University, Cairo, Egypt
}

Received 24 December 2013

Accepted 19 May 2014

\begin{abstract}
In this paper, the problem of testing exponentiality against used better than aged in convex ordering classes of life distributions is investigated. For this property a nonparametric test is presented based on kernel method. The test is presented for complete and right censored data. Furthermore, Pitman's asymptotic relative efficiency (PARE) is discussed to assess the performance of the test with respect to other tests. Selected critical values are tabulated. Some numerical simulations on the power estimates are presented for proposed test. Finally, examples in medical sciences are used as practical applications for the proposed test.
\end{abstract}

Keywords: UBAC Classes of Life Distributions; Kernel Method; Survival function; Right censored data; Kaplan-Meier estimator.

\section{Introduction}

During the past decades, various classes of life distributions have been proposed in order to model different aspects of aging. The best known of these classes are IFR, IFRA, DMRL, NBU, NBUE, and HNBUE. Properties and applications of these aging notions can be found, in Bryson and Siddiqui [6], Barlow and Proschan [4], Rolski [10], Klefsjö [9], and Stoyan [12].

Let $\mathrm{X}$ be a random variable describing the life time of a brand new device which begins to work at time $t=0$. As usual in the reliability, we denoted by $X_{t}$ the life time of 
the device of age $t$ with $t \geq 0$. The probability that the device of age $t$ still working till time $x$ (the survival function) is, $\bar{F}_{t}(x)=P[X>x+t \mid x>t]=\bar{F}(x+t) / \bar{F}(t)$, where $\bar{F}(x)$ is the survival function of $X$. Some properties concerning the asymptotic behavior of $X_{t}$ as $t \rightarrow \infty$ will be used.

Definition (1.1): (Bhattacharjee, [5]), If $\mathrm{X}$ is a nonnegative random variable, its distribution function $\mathrm{F}(\mathrm{x})$ is said to be finitely and positively smooth if a number $\gamma \quad(0$, $\infty$ ) exists such that $\lim _{t \rightarrow \infty} \bar{F}_{t}(x)=e^{-\imath x}$ for all $x \geq 0$, where $\gamma$ will be called the asymptotic decay coefficient of $X$. Denoting $X_{e}$ be an exponentially distributed random variable with mean $1 / \gamma$, the following definitions implies that $X_{t}$ converges to $X_{e}$ in distribution written as $X_{t} \stackrel{D}{\longrightarrow} X_{e}$. This property is useful for description of random life times of devices of unknown age.

Definition (1.2): The distribution function $F$ is said to be used better than age (UBA) if for all $x, t \geqslant 0 ; \quad \bar{F}_{t}(x) \geq e^{-\gamma x}$ or $\bar{F}(x+t) \geq \bar{F}(t) e^{-\gamma x}$.

From definition (1.2), we have the following definition:

Definition (1.3): The distribution function $F$ is said to be used better than aged in convex ordering (UBAC) if for all $x, t \geqslant 0$

$$
\int_{x}^{\infty} \bar{F}(u+t) d u \geq \bar{F}(t) \int_{x}^{\infty} e^{-\gamma u} d u \quad \text { or } v(x+t) \geq \frac{1}{\gamma} \bar{F}(t) e^{-\gamma x}
$$

Where: $v(x+t)=\int_{x+t}^{\infty} \bar{F}(u) d u$.

We observe that the inequality of (1.2) is achieved when $\mathrm{F}(\mathrm{x})$ has an exponential distribution with mean $\mu$ equal to the coefficient of the asymptotic decay $\gamma$, where the exponential distribution is the only one which has the lack of memory property. Willmot and Cai [13] showed that the UBA class includes the DMRL class. While Al - Nachawati and Alwasel [2] showed that UBAC class includes the UBA class of life distribution. Thus, we have IFR DMRL UBA UBAC.

Testing exponentiality against the classes of life distribution based on kernel method has seen a good deal of attention. For testing against IFR and DMRL, we refer Ahmed [1]. Our goal in this paper is to propose a new nonparametric test for exponentiality against UBAC based on kernel method in section 2. We use Pitman's asymptotic relative efficiency (PARE) to assess the performance of the test and make a comparison with other available tests, in section 3. A nonparametric test is presented for right censored data in section 4. Finally, examples using data from Attia et al [3] in medical science is given in section 5 . 


\section{Testing Exponentiality Versus UBAC Class Based On Kernel Method}

Suppose the lifetime $\mathrm{X}$ of a component has a distribution function $F$ which is unknown to us. Available to us are independent observations on $n$ components; i.e. we have at our disposal a random sample $X_{1}, X_{2}, \ldots, X_{n}$ from the distribution $F$. We have a null hypothesis $H_{0}$ and its alternative $H_{1}$, where $H_{0}: \mathrm{F}$ is exponential versus $H_{1}: F$ belongs to the class UBAC and $\mathrm{F}$ is not exponential.

Ismail and Abu-Youssef [7] introduced testing exponentiality versus UBAC class based on U-statistic but in this paper, we suggest testing exponentiality versus UBAC class of life distribution based on kernel method as follow:

$$
\delta_{K}=\int_{0}^{\infty} \int_{0}^{\infty} f(x)\left[\frac{1}{\gamma} \bar{F}(t) e^{-\gamma x}-v(x+t)\right] d F(x) d F(t) .
$$

It is easy to see that if $F$ is exponential, then $\delta_{K}=0$, while under $H_{1}$, we have $\delta_{K}>(<) 0$. Since the distribution function $\mathrm{F}$ is unknown to us, as a consequence we do not know the $\delta_{K}$. This means we need to construct $\hat{\delta}_{K}$ a good estimator of $\delta_{K}$. This can be done in terms of $F_{n}$, the empirical distribution function based on the available sample $X_{1}, X_{2}, \ldots, X_{n}$ from $\mathrm{F}$.

The estimator $\hat{\delta}_{K}$ of our test statistic is defined by

$$
\hat{\delta}_{K}=\int_{0}^{\infty} \int_{0}^{\infty} \hat{f}_{n}(x)\left[\frac{1}{\gamma} \hat{\bar{F}}_{n}(t) e^{-\hat{\gamma} x}-\hat{v}_{n}(x+t)\right] d F_{n}(x) d F_{n}(t) \text {. }
$$

As usual we denoted by $X_{(1)}, X_{(2)}, \ldots, X_{(n)}$ the corresponding ordered sample and if $\hat{F}_{n}$ is the empirical distribution function, then $\hat{F}_{n}(x)=\frac{1}{n} \sum_{j=1}^{n} I\left(X_{j}>x\right)$ is the empirical survival function, and $F_{n}(x)=\frac{i}{n}$ for $x \in\left[X_{(i)}, X\right)$, where $\mathrm{i}=1,2, \ldots, \mathrm{n}$.

In order to suggest an estimator for $\delta_{K}$ we first construct estimators $\hat{v}_{n}(x), \hat{\gamma}$ and $\hat{f}_{n}(x)$ for $v(x), \gamma$ and $f(x)$ respectively. $\hat{v}_{n}(x)=\frac{1}{n} \sum_{i=1}^{n}\left(X_{i}-x\right) I\left(X_{i}>x\right)$, $\hat{\gamma}=\frac{n}{\sum_{i=1}^{n} x_{i}}$ and $\hat{f}_{n}(x)=\frac{1}{n a_{n}} \sum_{l=1}^{n} K\left(\frac{x-X_{l}}{a_{n}}\right)$ where $I\left(X_{i}>x\right)$ is the indicator function; $I\left(X_{i}>x\right)=1$, if $X_{i}>x$; otherwise $I\left(X_{i}>x\right)=0$. and $K($.$) be a known probability$ density function, symmetric and bounded with mean zero and finite variance $\sigma_{K}^{2}$. Let $a_{n}$ be a sequence of real such that $a_{n} \rightarrow 0$ and $n a_{n} \rightarrow \infty, n \rightarrow \infty$.

These properties suggest writing the following estimator as 


$$
\hat{\delta}_{K}=\frac{1}{n^{4} a_{n}} \sum_{i=1}^{n} \sum_{j=1}^{n} \sum_{k=1}^{n} \sum_{l=1}^{n} K\left(\frac{X_{i}-X_{l}}{a_{n}}\right)\left[\frac{1}{\hat{\gamma}} e^{-\hat{\gamma} X_{j}}\left(X_{i}>X_{k}\right)-\left(X_{i}-X_{j}-X_{k}\right) I\left(X_{i}>X_{j}+X_{k}\right)\right]
$$

To make the test statistic in scale invariant, we take $\hat{\delta}_{K}^{*}=\frac{\hat{\delta}_{K}}{\bar{X}}$.

In order to use the U-statistic procedure, we set

$$
\varphi\left(X_{1}, X_{2}, X_{3}, X_{4}\right)=K\left(\frac{X_{1}-X_{4}}{a_{n}}\right)\left[\frac{1}{\hat{\gamma}} e^{-\hat{\gamma} X_{2}} I\left(X_{1}>X_{3}\right)-\left(X_{1}-X_{2}-X_{3}\right) I\left(X_{1}>X_{2}+X_{3}\right)\right]
$$

Here $X_{1}, X_{2}, X_{3}$ and $X_{4}$ are four independent lifetimes each with distribution function $\mathrm{F}$. We define the symmetric kernel as

$$
\phi\left(X_{1}, X_{2}, X_{3}, X_{4}\right)=\frac{1}{4 !} \sum_{R} \varphi\left(X_{1}, X_{2}, X_{3}, X_{4}\right)
$$

Where the sum is over all permutations of $X_{1}, X_{2}, X_{3}$ and $X_{4}$. Then $\hat{\delta}_{K}$ is equivalent to the U-statistic

$$
U_{n}=\frac{1}{\left(\begin{array}{l}
n \\
4
\end{array}\right)} \sum_{i<j<k<l} \varphi\left(X_{1}, X_{2}, X_{3}, X_{4}\right)
$$

Our test is rejects for large value of $U_{n}$.

Theorem (2.1): If $n^{4} a_{n} \rightarrow 0$ as $n \rightarrow \infty, \sqrt{n}\left(\hat{\delta}_{K}^{*}-\delta_{K}^{*}\right)$ is asymptotically normal with mean 0 and variance $V^{2}$ where,

$$
\begin{aligned}
V^{2}=\operatorname{Var} & {\left[2 f\left(X_{1}\right)\left[\frac{1}{\gamma} \int_{0}^{\infty} \int_{0}^{u} e^{-\gamma u} d F(y) d F(u)-\int_{0}^{X_{1} X_{1}-y} \int_{0}^{X_{1}}\left(X_{1}-u-y\right) d F(u) d F(y)\right]\right.} \\
+ & \frac{1}{\gamma} \int_{0}^{\infty} \int_{0}^{u} e^{-\gamma X_{1}} f(u) d F(y) d F(u)-\int_{0}^{X_{0}} \int_{X_{1}-u}^{0}\left(u-X_{1}-y\right) f(u) d F(y) d F(u) \\
+ & \left.\frac{1}{\gamma} \int_{0}^{\infty} \int_{0}^{X_{1}} e^{-\gamma y} f(u) d F(y) d F(u)-\int_{X_{1}}^{u-X_{1}} \int_{0}^{1}\left(u-X_{1}-y\right) f(u) d F(y) d F(u)\right]
\end{aligned}
$$

Proof: We use theorem of Serfling [11], which states that if $c_{1}<\infty$, then $\sqrt{n}\left(U_{n}-\theta\right) \rightarrow N\left(o, m^{2} c_{1}\right)$, where $c_{1}=\operatorname{Var}\left[\phi\left(X_{1}\right)\right]$. we need to find $\phi\left(X_{1}\right)$ which is by definition

$$
\begin{aligned}
\phi\left(X_{1}\right)= & E\left[\varphi\left(X_{1}, X_{2}, X_{3}, X_{4}\right) \mid X_{1}\right]+E\left[\varphi\left(X_{2}, X_{1}, X_{3}, X_{4}\right) \mid X_{1}\right] \\
& +E\left[\varphi\left(X_{2}, X_{3}, X_{1}, X_{4}\right) \mid X_{1}\right]+E\left[\varphi\left(X_{2}, X_{3}, X_{4}, X_{1}\right) \mid X_{1}\right]
\end{aligned}
$$

This can be written explicitly as follows:

$$
E\left[\varphi\left(X_{1}, X_{2}, X_{3}, X_{4}\right) \mid X_{1}\right]=f\left(X_{1}\right)\left[\frac{1}{\gamma} \int_{0}^{\infty} \int_{0}^{u} e^{-\gamma u} d F(y) d F(u)-\int_{0}^{X_{1} X_{1}-y} \int_{0}^{1}\left(X_{1} u-y\right) d F(u) d F(y)\right.
$$

Similarly, we have 
$E\left[\varphi\left(X_{2}, X_{1}, X_{3}, X_{4}\right) \mid X_{1}\right]=\frac{1}{\gamma} \int_{0}^{\infty} \int_{0}^{u} e^{-\gamma X_{1}} f(u) d F(y) d F(u)-\int_{0}^{X_{1}} \int_{X_{1}-u}^{0}\left(u-X_{1}-y\right) f(u) d F(y) d F(u)$

and

$E\left[\varphi\left(X_{2}, X_{3}, X_{1}, X_{4}\right) \mid X_{1}\right]=\frac{1}{\gamma} \int_{0}^{\infty} \int_{0}^{X_{1}} e^{-\gamma y} f(u) d F(y) d F(u)-\int_{X_{1}}^{0} \int_{0}^{u-X_{1}}\left(u-X_{1}-y\right) f(u) d F(y) d F(u)$

Observe that $E\left[\varphi\left(X_{2}, X_{3}, X_{1}, X_{4}\right) \mid X_{1}\right]$ has the same representation as (2.6). By taking the variance of $\phi\left(X_{1}\right)$ we get $c_{1}$.

Corollary (2.1): If the null hypothesis $H_{0}$ is true, i. e., the lifetime distribution $\mathrm{F}$ is exponential, then as we mentioned before, $\delta_{K}=0$ and we calculate explicitly the null variance, $V_{0}^{2}=0.387$.

Proof: Under $H_{0}, \bar{F}_{0}=\bar{F}(x)=e^{-x}$ and by direct calculation we found

$$
\phi_{0}\left(X_{1}\right)=-\frac{1}{12}\left[6\left(X_{1}-7 e^{-X_{1}}\right)+\left(24 X_{1}+37\right) e^{-2 X_{1}}\right]
$$

Thus, $\mathrm{E}\left[\phi_{0}\left(X_{1}\right)\right]=0$ and $\operatorname{Var}\left[\phi_{0}\left(X_{1}\right)\right]=c_{1}=0.387$.

To conduct the test, calculate $\sqrt{n} \hat{\delta}_{K} / V_{0}$ and reject $H_{0}$ if this value exceeds the standard normal value $Z_{1-\alpha}$. To illustrate the test, we have simulated the upper percentile points for the significance level $\alpha=0.01,0.02$, and 0.05 . The calculation of the test $\hat{\delta}_{K}$ is based on 10000 simulated samples from the standard exponential distribution. Table (1), gives the critical values of the test statistic $\hat{\delta}_{K}$. Figure (1) shows the critical values of the test statistic $\hat{\delta}_{K}$ are decreasing as the sample size increasing as follow: 
Table (1): Critical value for $\hat{\delta}_{K}$

\begin{tabular}{|c|c|c|c|}
\hline $\mathrm{n}$ & $95 \%$ & $98 \%$ & $99 \%$ \\
\hline 4 & 0.607514 & 0.722601 & 0.800363 \\
\hline 6 & 0.50763 & 0.601598 & 0.66509 \\
\hline 8 & 0.431089 & 0.512468 & 0.567453 \\
\hline 10 & 0.385577 & 0.458365 & 0.507546 \\
\hline 12 & 0.351982 & 0.418428 & 0.463324 \\
\hline 14 & 0.325872 & 0.387389 & 0.428954 \\
\hline 16 & 0.304826 & 0.362369 & 0.40125 \\
\hline 18 & 0.290954 & 0.345206 & 0.381863 \\
\hline 20 & 0.276294 & 0.327763 & 0.362539 \\
\hline 22 & 0.259956 & 0.30903 & 0.342187 \\
\hline 24 & 0.247201 & 0.294185 & 0.325931 \\
\hline 26 & 0.239125 & 0.284266 & 0.314767 \\
\hline 28 & 0.230427 & 0.273925 & 0.303317 \\
\hline 30 & 0.222613 & 0.264637 & 0.293032 \\
\hline 32 & 0.231136 & 0.271826 & 0.299319 \\
\hline 34 & 0.207966 & 0.24744 & 0.274112 \\
\hline 36 & 0.203217 & 0.24158 & 0.26750 \\
\hline 38 & 0197797 & 0.235136 & 0.260365 \\
\hline 40 & 0.194296 & 0.23069 & 0.25528 \\
\hline 42 & 0.195178 & 0.230694 & 0.254692 \\
\hline 44 & 0.180863 & 0.215563 & 0.239009 \\
\hline 46 & 0.179776 & 0.213714 & 0.236644 \\
\hline 48 & 0.175991 & 0.209214 & 0.231662 \\
\hline 50 & 0.176895 & 0.209447 & 0.231441 \\
\hline & & & \\
\hline
\end{tabular}

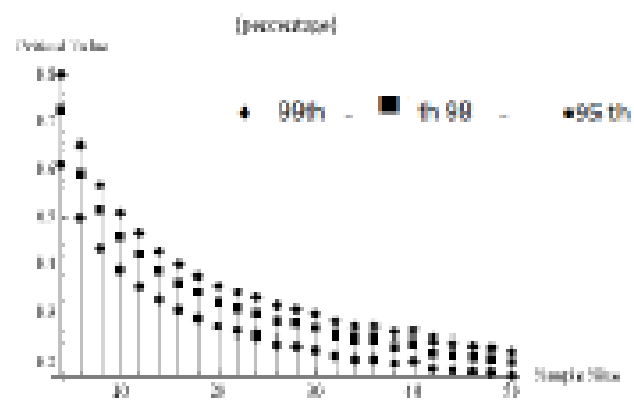

Figure 1: The Relation Between Sample Size and Critical Values 
The power estimate of the test statistic $\hat{\delta}_{K}$ is useful in clarifying how much the test can detect the departure from exponentiality towards the class UBAC. The higher value of the power estimate indicates that the test statistic is more able to detect such a departure. The power of the test statistics $\hat{\delta}_{K}$ is considered for $5 \%$ percentile in Table (2) for three alternatives. These alternatives are:

1. Linear failure rate: $\bar{F}_{\theta}(x)=e^{-\left(x+\frac{1}{2} \theta x^{2}\right)}, x>0, \theta \geq 0$

2. Makeham: $\bar{F}_{\theta}(x)=e^{-x-\theta\left(x-1+e^{-x}\right)}, x>0, \theta \geq 0$

3. Weibull: $\bar{F}_{\theta}(x)=e^{-x^{\theta}} x>0, \theta \geq 0$

For appropriate values of $\theta$, these distributions can be reduced to the exponential distribution. The power estimate of the test statistic $\hat{\delta}_{K}$, given in Table (2) shows the chance of detecting departure from exponentiality towards the UBAC property as $\theta$ increases, or the sample size $\mathrm{n}$ increases for the linear failure rate, Makeham, and Weibull distribution.

Table (2): Power estimates for $\hat{\delta}_{K}$

\begin{tabular}{|l|l|l|l|l|}
\hline Distribution & $\theta$ & $\mathrm{n}=10$ & $\mathrm{n}=20$ & $\mathrm{n}=30$ \\
\hline \multirow{5}{*}{ Linear failure rate } & 2 & 0.995 & 0.999 & 0.999 \\
\cline { 2 - 5 } & 3 & 0.998 & 0.999 & 1 \\
\cline { 2 - 5 } & 4 & 0.999 & 1 & 1 \\
\hline \multirow{3}{*}{ Makeham } & 2 & 0.993 & 0.999 & 0.999 \\
\cline { 2 - 5 } & 3 & 0.995 & 0.999 & 0.999 \\
\cline { 2 - 5 } & 4 & 0.999 & 0.999 & 1 \\
\hline Weibull & 2 & 0.999 & 1 & 1 \\
\hline
\end{tabular}

\section{Asymptotic relative efficiency:}

In this section we compare the power of the test statistic $\hat{\delta}_{K}$ using the concept of Pitman's asymptotic efficiency (PARE). To do this we need to evaluate the Pitman's asymptotic efficiency (PAE) of our test $\hat{\delta}_{K}$ and compare the PAR of other test to get PARE. Let $F_{\theta_{n}}$ be a sequence of alternative distributions, where $\theta_{n}=\theta_{0}+k / \sqrt{n}$, $\mathrm{k}$ is positive number, and $\theta_{0}$ corresponds to the exponential distribution. PAE is given by $e_{F_{\theta}}\left(T_{n, 1}\right)=\left.\lim _{n \rightarrow \infty} \frac{d}{d \theta} E_{\theta}\left(T_{n, 1}\right)\right|_{\theta \rightarrow \theta_{0}}\left(\sigma_{0}\right)^{-1}$ where $\sigma_{0}^{2}=V_{0}^{2}$ is the null asymptotic variance.

The PARE of $T_{n, 1}$ with respect to another test $T_{n, 2}$ is then given by $e_{F_{\theta}}\left(T_{n, 1}\right) / e_{F_{\theta}}\left(T_{n, 2}\right)$. the efficiencies of $\hat{\delta}_{K}$ are calculated for the following alternatives: 
linear failure rate, Makeham, and weibull distributions. Direct calculation of asymptotic efficiencies of the test $U_{n}$ and $\hat{\delta}_{K}$ for NBUE, Kanjo [8] and UBAC classes respectively are summarized in Table (3). In Table (4) we give efficiencies of $\hat{\delta}_{K}$ with respect to $U_{n}$. These calculations clearly indicate that the test proposed in this paper is well comparable with other test widely used in practice, and in some cases is even better.

Table (3): PAR of $\hat{\delta}_{K}$ and $U_{n}$

\begin{tabular}{|l|l|l|}
\hline Distribution & $\hat{\delta}_{K}$ & $U_{n}$ \\
\hline Linear failure rate & 0.565 & 0.433 \\
\hline Makeham & 0.245 & 0.144 \\
\hline Weibull & 0.424 & 0.132 \\
\hline
\end{tabular}

Table (4): PARE of $\hat{\delta}_{K}$ with respect to $U_{n}$

\begin{tabular}{|l|l|l|l|}
\hline Relative efficiency & Linear failure rate & Makeham & Weibull \\
\hline$e_{F_{\theta}}\left(T_{n, 1}\right) / e_{F_{\theta}}\left(T_{n, 2}\right)$. & 1.305 & 1.701 & 3.121 \\
\hline
\end{tabular}

\section{Testing against UBAC class for right censored data.}

In this section, a test statistic proposed to test $H_{0}$ versus $H_{1}$ with randomly right censored samples. In the censored model, instead of dealing with $X_{1}, X_{2}, \ldots, X_{n}$, we observe the pair $\left(Z_{i}, \delta_{i}\right), \mathrm{i}=1,2, \ldots, \mathrm{n}$, where $Z_{i}=\min \left(X_{i}, Y_{i}\right)$ and $\delta_{i}=1$ if $Z_{i}=X_{i}$, $\delta_{i}=0$ if $Z_{i}=Y_{i}$, where $X_{1}, X_{2}, \ldots, X_{n}$ denote their true life time from a distribution $\mathrm{F}$ and $Y_{1}, Y_{2}, \ldots, Y_{n}$ be i.i.d. according to distribution G. Also $X$ 's and $Y^{\prime}$ 's are independent. Let $Z_{(0)}=0 \leq Z_{(1)} \leq Z_{(2)} \leq \ldots \leq Z_{(n)}$ denote the order $Z$ 's and $\delta_{(i)}$ is the $\delta_{i}$ corresponding to $Z_{(i)}$, respectively. Using the Kaplan Meier estimator in the case of censored data $\left(Z_{i}, \delta_{i}\right), \mathrm{i}=1 \ldots \mathrm{n}$, the proposed test statistic for right censored data is given by

$$
\hat{\delta}_{K}^{c}=\sum_{i=1}^{n} \sum_{j=1}^{n} \hat{f}(x)\left[e^{i Z_{(j)}} \hat{\bar{F}}_{n}(t)-\hat{v}_{n}(x+t)\right]\left[\prod_{p=1}^{i-2} C_{p}^{\delta_{i}}-\prod_{p=1}^{i-1} C_{i}^{\delta_{i}}\right]\left[\prod_{q=1}^{j-2} C_{j}^{\delta_{j}}-\prod_{q=1}^{j-1} C_{j}^{\delta_{j}}\right]
$$

Where: $\hat{v}_{n}(x+t)=\int_{x+t}^{\infty} \hat{\bar{F}}_{n}(z) d z=\hat{\mu}-\int_{0}^{x+t} \hat{\bar{F}}_{n}(z) d z$

$$
=\hat{\mu}-\sum_{k=1}^{l} \prod_{m=1}^{k-1} C_{m}^{\delta_{m}}\left(Z_{(k)}-Z_{(k-1)}\right)
$$

Where, $l=i+j$ if $Z_{(i)}+Z_{(j)}<Z_{(n)}, 1=\mathrm{n}$ if $Z_{(i)}+Z_{(j)}>Z_{(n)}$ 


$$
\begin{aligned}
& \hat{\mu}=\sum_{j=1}^{l} \prod_{k=1}^{j-1} C_{k}^{\delta_{k}}\left(Z_{(j)}-Z_{(j-1)}\right) \\
& d \hat{F}_{n}\left(Z_{j}\right)=\left[\prod_{q=1}^{j-2} C_{q}^{\delta_{q}}-\prod_{q=1}^{j-1} C_{q}^{\delta_{q}}\right] \\
& \hat{f}(x)=\sum_{m=1}^{n} \delta_{m} K\left(x-Z_{(k)}\right)
\end{aligned}
$$

and

$\hat{\bar{F}}_{n}(t)=\prod_{m<Z_{(m)}<t} C_{m}^{\delta_{m}}$ Where $C_{m}=\frac{n-m}{n-m+1}$ and $t \in\left[0, Z_{(m)}\right]$.

To illustrate the test, we have simulated the upper percentile points for the significance level $\alpha=0.01,0.02$, and0.05. The calculation of the test $\hat{\delta}_{K}^{C}$ is based on 10000 simulated samples from the standard exponential distribution. Table (5), gives the critical values of the test statistic $\hat{\delta}_{K}^{C}$. Figure (2) shows the critical values of the test statistic $\hat{\delta}_{K}^{C}$ are decreasing as the sample size increasing as follow:

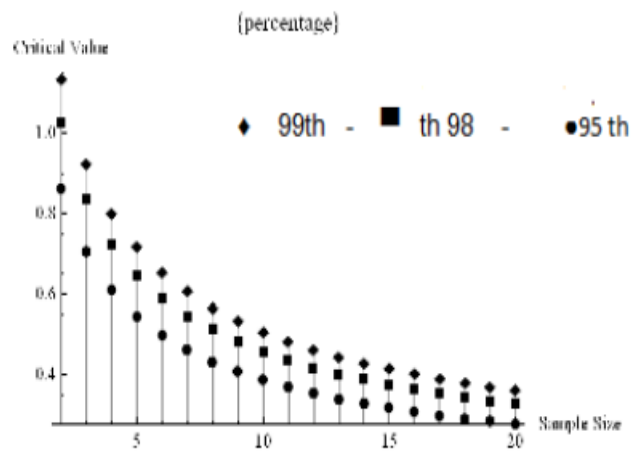

Figure 2: The Relation Between Sample Size and Critical Values (Censored Data) 
Table (5): Critical value of $\hat{\delta}_{K}^{C}$

\begin{tabular}{|l|l|l|l|}
\hline $\mathrm{n}$ & $95 \%$ & $98 \%$ & $99 \%$ \\
\hline 1 & 0.862262 & 1.025020 & 1.13499 \\
\hline 3 & 0.704056 & 0.836947 & 0.926738 \\
\hline 4 & 0.609749 & 0.724836 & 0.802598 \\
\hline 5 & 0.545394 & 0.648331 & 0.717883 \\
\hline 6 & 0.497891 & 0.591860 & 0.655352 \\
\hline 7 & 0.460975 & 0.547973 & 0.606755 \\
\hline 8 & 0.431221 & 0.512600 & 0.567586 \\
\hline 9 & 0.406579 & 0.483304 & 0.535145 \\
\hline 10 & 0.385736 & 0.458524 & 0.507704 \\
\hline 11 & 0.367808 & 0.437209 & 0.484101 \\
\hline 12 & 0.352176 & 0.418622 & 0.463518 \\
\hline 13 & 0.338390 & 0.402229 & 0.445363 \\
\hline 14 & 0.326116 & 0.387633 & 0.429198 \\
\hline 15 & 0.315099 & 0.374530 & 0.414686 \\
\hline 16 & 0.305144 & 0.362687 & 0.401568 \\
\hline 17 & 0.296095 & 0.351921 & 0.389640 \\
\hline 18 & 0.287833 & 0.342085 & 0.378742 \\
\hline 19 & 0.280262 & 0.33068 & 0.368747 \\
\hline 20 & 0.273316 & 0.324785 & 0.359561 \\
\hline 51 & 0.175919 & 0.208150 & 0.229928 \\
\hline 61 & 0.157073 & 0.186544 & 0.206457 \\
\hline 71 & 0.145133 & 0.172450 & 0.190907 \\
\hline 81 & 0.135724 & 0.161299 & 0.178580 \\
\hline 91 & 0.127978 & 0.152107 & 0.168411 \\
\hline 101 & 0.121439 & 0.144342 & 0.159817 \\
\hline & & & \\
\hline
\end{tabular}

\section{Application:}

\subsection{Application for complete data:}

Example (1): The following data represent 39 liver cancers patients taken from El Minia Cancer Center Ministry of Health Egypt Attia et al [3] the ordered life times (in days) are:

$10 ; 14 ; 14 ; 14 ; 14 ; 14 ; 15 ; 17 ; 18 ; 20 ; 20 ; 20 ; 20 ; 20 ; 23 ; 23 ; 24 ; 26 ; 30 ; 30$; $31 ; 40 ; 49 ; 51 ; 52 ; 60 ; 61 ; 67 ; 71 ; 74 ; 75 ; 87 ; 96 ; 105 ; 107 ; 107 ; 107 ; 116 ; 150$ : It was found that the test statistic for the data set, $\hat{\delta}_{K}=235.999$, which it exceeds the critical value of table 3 . Then we reject the null hypothesis of exponentiality.

\subsection{Application for censored data:}

Example (2): The following data represent 39 liver cancers patients taken from El Minia Cancer Center Ministry of Health Egypt Attia et al [3] the ordered life times (in days) are: 
(i) Non-censored data

$10 ; 14 ; 14 ; 14 ; 14 ; 14 ; 15 ; 17 ; 18 ; 20 ; 20 ; 20 ; 20 ; 20 ; 23 ; 23 ; 24 ; 26 ; 30 ; 30$;

$31 ; 40 ; 49 ; 51 ; 52 ; 60 ; 61 ; 67 ; 71 ; 74 ; 75 ; 87 ; 96 ; 105 ; 107 ; 107 ; 107 ; 116 ; 150$.

(ii) Censored data

$30 ; 30 ; 30 ; 30 ; 30 ; 60 ; 150 ; 150 ; 150 ; 150 ; 150 ; 185$ :

It was found that the test statistic for the data set, $\hat{\delta}_{K}^{C}=0.170761$, which it decreases the critical value of table 3 . Then we accept the null hypothesis of exponentiality.

Acknowledgement: The author thanks very much the referee for his (her) comments and corrections.

\section{References:}

[1] Ahmad, I. A. (2000). Testing exponentiality against positive ageing kernel methods. The Indian Journal of Statistics, 62, 244-257.

[2] Al-Nachawati, H. and Al-wasal, I. A. (1997). On used better than aged in convex ordering class of life distributions. Journal of statistical research, 31(1), 123-130.

[3]Attia, A. F., Mahmoud, M. A. W. and Abdul-Moniem, I. B. (2004). On testing for exponential better than used in average class of life distributions based on the Utest. The proceeding of the 39 th annual conference on statistics, SR Cairo university-Egypt, 11-14.

[4] Barlow, R. E. and Proschan, F. (1981). Statistical theory of reliability and life testing probability models. To begin with, silver spring, MD.

[5] Bhattacharjee, M. (1982). The class of mean residual and some consequences. SIAM journal on algebraic and discrete methods, 56-65.

[6] Bryson, M. C. and Siddiqui, M. M. (1969). Some criteria for aging. Journal of the American Statistical Association, 64, 1472-1483.

[7] Ismail, A. A. and Abu-Youssef, S. E. (2012). A goodness of fit approach to class of life distributions with unknown age. Quality and reliability engineering international, 28(7), 761-766.

[8] Kanjo, A. I. (1993). An exact test for NBUE class of survival functions. Commun. Statist. Theory and Methods, 22(3), 787-795.

[9] Klefsjö, B. (1982). HNBUE and HNWUE classes of life distributions. Nav Res Logistics Quart, 29, 419-422.

[10] Rolski, T. (1975). Mean residual life. Bull Int Stat Inst., 46, 266-270.

[11] Serfling, R. J. (1980). Approximation Theorems of Mathematical Statistics. Wiley, New York.

[12] Stoyan, D. (1983). Comparison methods for queues and other stochastic models. Wiley Interscience, New York.

[13] Willmot, G. and Cai, J. (2000). On classes of life time distributions with unknown age. Probability in the engineering and informational sciences, 14, 473484. 